\title{
Patients' experience of shoulder disorders: a systematic review of qualitative studies for the OMERACT Shoulder Core Domain Set
}

\author{
Matthew J. Page ${ }^{1}$, Denise A. O'Connor ${ }^{2,3}$, Mary Malek ${ }^{4}$, Romi Haas ${ }^{2,3}$, \\ Dorcas Beaton $^{5}$, Hsiaomin Huang ${ }^{6}$, Sofia Ramiro ${ }^{7,8}$, Pamela Richards ${ }^{9}$, \\ Marieke J. H. Voshaar ${ }^{10}$, Beverley Shea ${ }^{11,12}$, Arianne P. Verhagen ${ }^{13}$, \\ Samuel L. Whittle ${ }^{14}$, Danielle A. van der Windt ${ }^{15}$, Joel J. Gagnier ${ }^{6,16}$ and \\ Rachelle Buchbinder ${ }^{2,3}$; for the OMERACT Shoulder Core Set Working Group
}

\begin{abstract}
Objectives. To describe the experiences (including symptoms and perceived impacts on daily living) of people with a shoulder disorder.

Methods. Systematic review of qualitative studies. We searched for eligible qualitative studies indexed in Ovid MEDLINE, Ovid Embase, CINAHL (EBSCO), SportDiscus (EBSCO) and Ovid PsycINFO up until November 2017. Two authors independently screened studies for inclusion, appraised their methodological quality using the Critical Appraisal Skills Programme checklist, used thematic synthesis methods to generate themes describing the experiences reported by participants and assessed the confidence in the findings using the Grading of Recommendations Assessment, Development and Evaluation Confidence in Evidence from Reviews of Qualitative research (GRADE-CERQual) approach.

Results. The inclusion criteria were met by eight studies, which included 133 participants (49 females and 84 males) with either rotator cuff disease, adhesive capsulitis, proximal humeral fracture, shoulder instability or unspecified shoulder pain. We generated seven themes to describe what people in the included studies reported experiencing: pain; physical function/activity limitations; participation restriction; sleep disruption; cognitive dysfunction; emotional distress; and other pathophysiological manifestations (other than pain). There were interactions between the themes, with particular experiences impacting on others (e.g. pain leading to reduced activities and sleep disruption). Following grading of the evidence, we considered it likely that most of the review findings were a reasonable representation of the experiences of people with shoulder disorders.

Conclusion. Patients with shoulder disorders contend with considerable disruption to their life. The experiences described should be considered by researchers seeking to select the most appropriate outcomes to measure in clinical trials and other research studies in people with shoulder disorders.
\end{abstract}

Key words: shoulder pain, qualitative research, systematic review, qualitative evidence synthesis, outcome assessment

${ }^{1}$ School of Public Health and Preventive Medicine, Monash University,
Melbourne, ${ }^{2}$ Monash Department of Clinical Epidemiology, Cabrini
Institute, Malvern, ${ }^{3}$ Department of Epidemiology and Preventive
Medicine, School of Public Health and Preventive Medicine, Monash
University, Melbourne, ${ }^{4}$ Monash University, Clayton, Victoria,
Australia, Institute of Health \& Work and the University of Toronto,
Toronto, Ontario, Canada, ${ }^{6}$ Department of Orthopaedic Surgery,
University of Michigan, Ann Arbor, MI, USA, ${ }^{7}$ Department of
Rheumatology, Leiden University Medical Center, Leiden, ${ }^{8}$ Zuyderland
Medical Center, Heerlen, The Netherlands, ${ }^{9}$ Academic Rheumatology
Bristol, University of Bristol, Bristol, UK, ${ }^{10}$ Department of Psychology,
Health and Technology, University of Twente, Enschede, The
Netherlands, ${ }^{11}$ Ottawa Hospital Research Institute, Clinical
Epidemiology Program, ${ }^{12}$ School of Epidemiology and Public Health,
Faculty of Medicine, University of Ottawa, Ottawa, Ontario, Canada, ${ }^{13}$ University of Technology Sydney, Sydney, New South Wales, ${ }^{14}$ Rheumatology Unit, The Queen Elizabeth Hospital, Adelaide, South Australia, Australia, ${ }^{15}$ Arthritis Research UK Primary Care Centre, Institute for Primary Care and Health Sciences, Keele University, Keele, UK and ${ }^{16}$ Department of Epidemiology, School of Public Health, University of Michigan, Ann Arbor, MI, USA

Submitted 18 November 2018; accepted 27 January 2019

Correspondence to: Matthew J. Page, School of Public Health and Preventive Medicine, Monash University, 553 St Kilda Road,

Melbourne, Victoria, 3004, Australia.

E-mail: matthew.page@monash.edu 
- People with shoulder disorders contend with several disruptive experiences.

- Some experiences appear to impact on others (e.g. shoulder pain can lead to avoidance of particular activities and sleep disruption).

- Identifying the problems most important to people with shoulder disorders may improve patient-centred care.

\section{Introduction}

Shoulder disorders, including rotator cuff disease, adhesive capsulitis, glenohumeral $\mathrm{OA}$ and other conditions such as instability and humeral head fractures, affect a considerable proportion of the general population, with an estimated point prevalence ranging from 7 to $26 \%[1,2]$. Common experiences of people with shoulder disorders include pain that can lead to problems with performing daily activities, such as dressing and bathing [3, 4]. Such experiences should be reflected in outcome measures used to investigate the effectiveness of treatment options for shoulder disorders. However, the outcomes used to evaluate interventions for people with shoulder disorders are not consistently measured in randomized trials. For example, in our previous analysis of shoulder trials published between 1954 and 2015, we found that 90\% measured pain, $71 \%$ measured performance of activities of daily living and $6 \%$ measured participation in work [5]. This diversity in outcome measurement limits our ability to compare findings between studies and to synthesize data in meta-analyses.

The OMERACT Shoulder Core Set Working Group was established in 2015 to develop a core domain set for clinical trials of interventions for shoulder disorders [6]. A core domain set is an agreed minimum set of outcome domains (i.e. constructs such as pain or function) that should be measured and reported in all clinical trials for a particular health condition, which can help reduce the diversity in outcome measurement in future trials [7]. We have used several approaches to inform the development of a core domain set for shoulder disorders. As noted above, we examined what outcome domains (such as pain) have been measured in previous shoulder trials, to generate a list of potentially important domains [5, 8]. We also conducted an international Delphi study, in which patients, clinicians and researchers were asked to consider the domains identified from our previous literature review, judge the importance of each domain and identify any domains missing from the list [4]. Findings of this research were discussed at face-to-face stakeholder meetings prior to and during the OMERACT 2016 conference, where we sought consensus on a preliminary core domain set [9].

Since the OMERACT 2016 conference, greater emphasis has been placed on the value of qualitative research to inform the development of core domain sets $[10,11]$. Using qualitative methods to explore the lived experience and perspectives of people with shoulder disorders may identify additional important outcome domains that have not been measured in existing trials or considered within the Delphi process. It may also highlight areas that clinicians should consider, which may improve patient-centred care [12]. We are aware of several qualitative studies that have investigated the lived experience of people with shoulder disorders, but to our knowledge, there has been no attempt to synthesize the findings of these studies. Therefore, we conducted a systematic review of qualitative studies to address the following question: what are the experiences (including symptoms and perceived impacts on daily living) of people with a shoulder disorder?

\section{Methods}

We registered our systematic review in PROSPERO in November 2017 (CRD42017082628; a full protocol for the review was uploaded at the same time). We reported our systematic review according to the Enhancing Transparency in Reporting the synthesis of Qualitative research statement [13].

\section{Eligibility criteria}

We included any study in which the authors used qualitative methods (e.g. focus groups, interviews, nominal group techniques, participant observation) to explore the experiences and perceptions of people living with a shoulder disorder. Eligible shoulder disorders included rotator cuff disease (an umbrella term to classify disorders of the rotator cuff, including subacromial impingement syndrome, rotator cuff tendinopathy or tendinitis, partial or full thickness rotator cuff tear, calcific tendinitis and subacromial bursitis [14]), adhesive capsulitis, dislocation or shoulder instability, glenohumeral or acromioclavicular $\mathrm{OA}$, glenoid labrum pathologies, proximal humeral or humeral head fractures, or unspecified shoulder pain. We included full articles (i.e. not conference abstracts) written in English, Dutch, French, Chinese or German (languages spoken by the authors) that were published in peer-reviewed journals. We included mixed-methods studies (i.e. those that reported both quantitative and qualitative data) but only if the qualitative data could be separated from the quantitative data. We also included mixed-participant studies (i.e. those that included participants with a shoulder disorder or another musculoskeletal condition, such as back pain or neck pain) only if the data on participants with shoulder disorders could be separated from data on participants with other conditions. The primary outcomes of our review included the symptoms of people with shoulder disorders and the perceived impact of these symptoms on their daily lives, and the outcome(s) of most importance to patients, as elicited by qualitative research methods.

We excluded qualitative studies that enrolled participants with systemic inflammatory conditions, such as RA, hemiplegia causing secondary shoulder pain, or 
pain in the shoulder region as part of a complex myofascial neck/shoulder/arm pain condition (e.g. complex regional pain syndrome). We excluded qualitative studies of health professionals who treat people with shoulder disorders. We also excluded studies that used quantitative instruments to measure patient-reported outcomes (e.g. surveys asking dichotomous or Likert-style questions).

\section{Search methods}

We conducted a comprehensive search to seek all available studies, rather than seeking all available concepts until theoretical saturation was achieved. We searched the following bibliographic databases, all from inception until November 2017: Ovid MEDLINE, Ovid Embase, CINAHL (EBSCO), SportDiscus (EBSCO) and Ovid PsycINFO (see all search strategies in supplementary Table S1, available at Rheumatology online). To capture any papers that may have been missed by the searches, one author (M.J.P.) examined the references of included articles.

\section{Selection of studies}

Two review authors (M.J.P. and M.M.) independently screened all titles and abstracts yielded from the searches, and all full text articles considered relevant. All disagreements were resolved via discussion or by consultation with a third review author (R.B.) when necessary. We summarized the selection of studies using a Preferred Reporting Items for Systematic Reviews and Meta-analyses (PRISMA) flow diagram [15].

\section{Data collection}

Pairs of review authors (M.J.P. and D.A.O. or M.M.) independently extracted the following data from each included study using a standardized data collection form: lead author, year of publication, country, research question, sample size, participant characteristics [age and sex/ gender, type(s) of shoulder disorders], data collection method (e.g. focus group, interview), data analysis method (e.g. thematic analysis), themes and sub-themes relating to individual symptoms and their perceived impacts on daily living that were generated by the study authors, along with any supporting quotes and explanations of each theme. Disagreements were resolved via discussion or through inclusion of a third review author (R.B.) when necessary.

\section{Quality assessment}

Pairs of review authors (M.J.P. and R.H. or M.M.) independently assessed the quality of the included studies using the Critical Appraisal Skills Programme (CASP) checklist for qualitative studies [16]. All disagreements were resolved via discussion. The CASP checklist consists of 10 items, each of which includes multiple signalling questions to help users interpret the item (29 signalling questions in total). Following the method employed by Slade et al. [17], we constructed a summary table detailing the frequency of responses ('Yes' or 'No') to each signalling question. A narrative summary of the quality of the included studies is also provided. Summary scores or quality ratings were not generated as the CASP checklist does not have a scoring matrix and a cut-off point has not been established for ratings of the quality of qualitative studies.

\section{Data analysis}

We analysed study data using the methods of thematic synthesis outlined by Thomas and Harden [18]. We used an inductive approach to coding. In the first stage, we read each line of extracted text and derived codes based on the meaning and content of each extract (e.g. the line '... the pain is really quite excruciating when I go to bed ...' was coded as 'pain severe at night'). As each new study was coded, existing codes were reviewed and revised, and new codes were added, when necessary. Once all studies had been coded, all text that had a given code was reviewed to check for consistency of coding across the studies, thus ensuring the translation of concepts from one study to another. One review author (M.J.P.) coded all text line-by-line, and codes were verified by another review author (D.A.O.). Any disagreements were resolved via discussion.

In the next stage, similarities and differences between the codes were reviewed in order to generate themes describing the experiences of people with a shoulder disorder. For example, all codes relating to the intensity or severity of pain were grouped under a theme labelled 'pain'. The generation of themes was based closely on what was reported in the included studies (i.e. quotes from patients and interpretations offered by the study authors). Two review authors (M.J.P. and D.A.O.) generated themes independently, then discussed and finalized the themes in consultation with another review author (R.B.).

We drafted 'review findings' to summarize each theme describing the experiences of people with a shoulder disorder [19]. Two review authors (M.J.P. and R.H.) then independently assessed the confidence in each of the review findings using the Grading of Recommendations Assessment, Development and Evaluation Confidence in Evidence from Reviews of Qualitative research (GRADECERQual) approach [19]. This approach requires assessors to consider four components: the methodological limitations of the qualitative studies contributing to each review finding (based on the CASP assessments) [20], the coherence of data contributing to each review finding (i.e. how clear and cogent the fit is between the data from the primary studies and a review finding that synthesizes the data) [21], the adequacy of data supporting each review finding (i.e. the degree of richness and the quantity of data supporting a review finding) [22] and the relevance of the studies contributing to each review finding (i.e. the extent to which the body of data from the primary studies supporting a review finding is applicable to the context specified in the review question) [23]. After considering the four components, both review authors independently judged the overall confidence in each review finding (i.e. for each theme generated) as: high: highly likely that the 
review finding is a reasonable representation of the phenomenon of interest; moderate: likely that the review finding is a reasonable representation of the phenomenon of interest; low: possible that the review finding is a reasonable representation of the phenomenon of interest; or very low: unclear whether the review finding is a reasonable representation of the phenomenon of interest [19]. Disagreements in confidence ratings were resolved via discussion. We presented the review findings, our confidence judgement for each finding and an explanation of the judgement in a Summary of Qualitative Findings table.

\section{Patient involvement}

Two patient research partners (P.R. and M.J.H.V.) were involved in the interpretation of the results and writing up of the manuscript.

\section{Results}

In total, 3664 records were identified from the searches, of which we retrieved 61 for full text screening (Fig. 1). Inclusion criteria were met by eight studies [24-31]. Most $(n=41)$ of the 53 excluded studies were excluded because they were not qualitative studies. We excluded eight qualitative studies that included either participants with ineligible conditions, or mixed populations, where data on participants with shoulder disorders could not be separated from data on other participants [32-39] (supplementary Table S2, available at Rheumatology online). We also excluded four qualitative studies that explored patients' views about a particular intervention (e.g. manual therapy), rather than the symptoms and perceived impact of their shoulder disorder on daily living [40-43] (supplementary Table S2, available at Rheumatology online).

Across the eight included studies there were 133 participants (Table 1). Participants had diagnoses of rotator cuff disease (three studies), adhesive capsulitis (two studies), proximal humeral fracture, shoulder instability or unspecified shoulder pain (one study each). The studies were conducted in the UK (four studies), Canada (two studies), Finland or New Zealand (one study each). Most participants were at least 40 years old, whereas one study focused on younger athletes with shoulder instability (mean age 27 years). There were 49 females and 84 males. Data were gathered using semi-structured interviews in seven of the studies, whereas focus groups were conducted in one study.

The methodological quality of the included studies varied (Fig. 2; supplementary Table S3, available at Rheumatology online). Strengths that were observed in all studies included clearly stated objectives; clearly stated data collection methods; approval sought from an ethics committee; and sufficient, explicit data presented to support the findings. At least one negative response to a CASP signalling question was recorded in all but one study [30]. The quality of one study [24] was particularly problematic, as responses to 18 of the 29 CASP signalling questions were negative. The following limitations were identified in more than half of the studies: no critical examination of the researchers' own role, potential bias and influence during formulation of the research question, data collection, analysis and selection of data for presentation; no explanation as to why patients approached for participation were the most appropriate to provide access to the type of knowledge sought by the study; no justification for the setting of data collection; and no description of issues around informed consent or confidentiality (Fig. 2).

We generated seven themes to describe what people in the included studies reported experiencing; these included: pain; physical function/activity limitations; participation restriction; sleep disruption; cognitive dysfunction; emotional distress; and other pathophysiological manifestations (other than pain). Table 2 provides a summary of each review finding and Table 3 presents illustrative quotes reflecting each finding. Not all participants reported having all of the above experiences. For example, while pain, physical function/activity limitations and sleep disruption were experienced by most participants in the studies, fewer participants reported experiencing cognitive dysfunction and other pathophysiological manifestations (e.g. poor muscle strength).

We generated sub-themes for three themes that addressed multiple concepts (Table 2). For example, the 'participation restriction' theme consists of sub-themes on 'work disruption', 'limited recreation' and 'limited social interactions', reflecting the finding that some participants may experience problems in one or multiple aspects of life participation. In addition, we generated sub-themes for 'emotional distress' to reflect the various emotional experiences that can accompany a shoulder disorder. Many people in the included studies reported experiencing frustration because of their shoulder disorder, whereas a smaller subset reported symptoms of depression, anxiety and a perception that their suffering was hidden from the casual observer given the lack of outward signs of their shoulder disorder.

There were interactions between the themes describing the experiences of people with a shoulder disorder (Fig. 3). Pain emerged as the predominant symptom that was perceived to 'affect everything', that is, lead to difficulties with performing activities of daily living, engaging in work and recreation, and getting to and staying asleep. Each of these experiences were perceived by participants to have several follow-on effects. For example, many patients claimed that work disruption caused their emotional distress, including frustration and anxiety about their recovery. Also, some people perceived that sleep disruption resulted in them having less energy to perform activities of daily living and recreational activities, and aggravated their pain, the following day (Fig. 3).

We considered it likely that most of the review findings were a reasonable representation of the experiences of people with shoulder disorders, i.e. we had 'moderate confidence' in most of the review findings (Table 2; a more detailed GRADE-CERQual Qualitative Evidence Profile is available in supplementary Table S4, available 
FIG. 1 Flow diagram of identification, screening and inclusion of studies

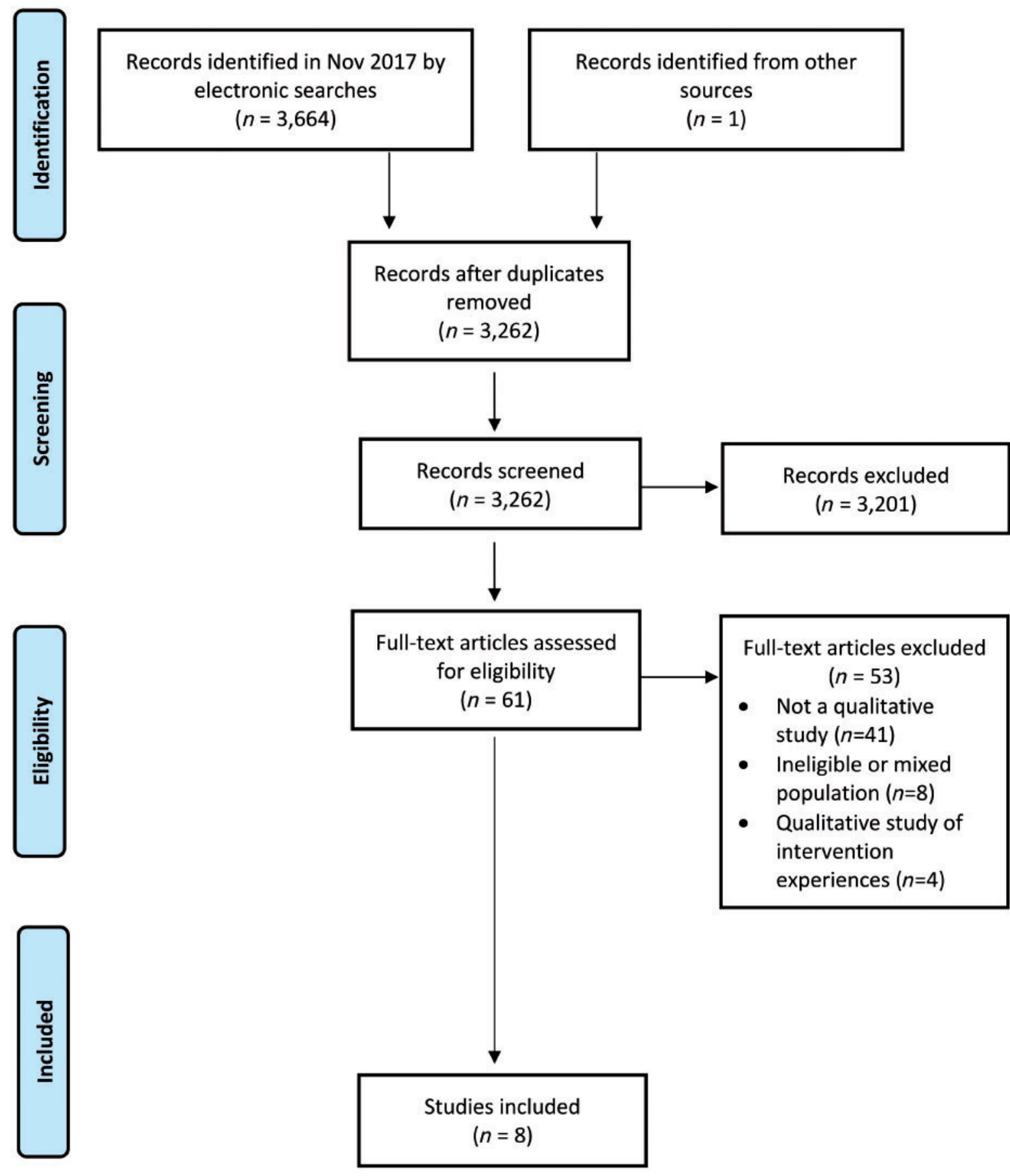

at Rheumatology online). Our judgement of 'moderate' rather than 'high' confidence was driven mostly by the minor concerns we had about methodological limitations of the contributing studies, and minor concerns about the relevance of each contributing study to the review question (given that all data came from only four high-income countries). In contrast, we had very low confidence in the finding that cognitive dysfunction is experienced by people with shoulder disorders, as this finding was based on limited data from two studies, one of which had several methodological limitations. We also had low confidence in the review findings regarding the perception of hidden suffering, and other pathophysiological manifestations, because of concerns about methodological limitations, adequacy of the data and relevance of studies to the review question. 
TABLE 1 Characteristics of included studies

\begin{tabular}{|c|c|c|c|c|c|c|}
\hline Study ID & $\begin{array}{l}\text { Shoulder } \\
\text { disorder }\end{array}$ & Country & $\begin{array}{l}\text { Sample } \\
\text { size }\end{array}$ & Gender & Age & Qualitative method \\
\hline $\begin{array}{l}\text { Carter } 2002 \\
\text { [24] }\end{array}$ & Adhesive capsulitis & UK & 20 & $10 \mathrm{~F}, 10 \mathrm{M}$ & $\begin{array}{c}75 \% \text { were aged } \\
\text { over } 50 \text { years }\end{array}$ & $\begin{array}{l}\text { Semi-structured } \\
\text { interview }\end{array}$ \\
\hline $\begin{array}{l}\text { Gillespie et al. } \\
2017 \text { [25] }\end{array}$ & $\begin{array}{l}\text { Rotator cuff-related } \\
\text { pain }\end{array}$ & New Zealand & 10 & $5 \mathrm{~F}, 5 \mathrm{M}$ & $\begin{array}{l}\text { Median } 58 \text { years } \\
\text { (range } 47-68)\end{array}$ & $\begin{array}{l}\text { Semi-structured } \\
\text { interview }\end{array}$ \\
\hline $\begin{array}{l}\text { Jones et al. } \\
2013 \text { [26] }\end{array}$ & Adhesive capsulitis & UK & 11 & $6 \mathrm{~F}, 5 \mathrm{M}$ & $\begin{array}{l}\text { All at least } 40 \text { years, } \\
\text { most }(n=7) \text { in } \\
\text { their } 50 \mathrm{~s}\end{array}$ & $\begin{array}{l}\text { Semi-structured } \\
\text { interview }\end{array}$ \\
\hline $\begin{array}{l}\text { Minns Lowe } \\
\text { et al. } 2014 \\
\text { [27] }\end{array}$ & Rotator cuff tear & UK & 20 & $4 \mathrm{~F}, 16 \mathrm{M}$ & $\begin{array}{l}\text { Mean } 67 \text { years } \\
\quad \text { (S.D. 6, range 54-75) }\end{array}$ & $\begin{array}{l}\text { Semi-structured } \\
\text { interview }\end{array}$ \\
\hline $\begin{array}{l}\text { Nyman et al. } \\
2012 \text { [28] }\end{array}$ & $\begin{array}{l}\text { Supraspinatus } \\
\text { tendinitis }\end{array}$ & Finland & 21 & $9 \mathrm{~F}, 12 \mathrm{M}$ & $\begin{array}{l}\text { Mean } 53 \text { years } \\
\text { (range 43-63) }\end{array}$ & Focus group \\
\hline $\begin{array}{l}\text { O'Hara et al. } \\
2017 \text { [29] }\end{array}$ & $\begin{array}{l}\text { Proximal humeral } \\
\text { fracture }\end{array}$ & Canada & 11 & $7 \mathrm{~F}, 4 \mathrm{M}$ & $\begin{array}{l}\text { Median } 68 \text { years } \\
\text { (range } 60-87 \text { ) }\end{array}$ & $\begin{array}{l}\text { Semi-structured } \\
\text { interview }\end{array}$ \\
\hline $\begin{array}{l}\text { Payne } 2010 \\
\text { [30] }\end{array}$ & $\begin{array}{l}\text { Unspecified } \\
\text { shoulder pain }\end{array}$ & UK & 15 & $7 \mathrm{~F}, 8 \mathrm{M}$ & $\begin{array}{l}\text { Mean } 48 \text { years (S.D. } 12 \text {, } \\
\text { range } 26-69 \text { ) }\end{array}$ & $\begin{array}{l}\text { Semi-structured } \\
\text { interview }\end{array}$ \\
\hline $\begin{array}{l}\text { Tjong et al. } \\
2015 \text { [31] }\end{array}$ & Shoulder instability & Canada & 25 & $1 \mathrm{~F}, 24 \mathrm{M}$ & $\begin{array}{l}\text { Mean } 27 \text { years } \\
\text { (S.D. 7) }\end{array}$ & $\begin{array}{l}\text { Semi-structured } \\
\text { interview }\end{array}$ \\
\hline
\end{tabular}

F: female; M: male.

FIG. 2 CASP methodological assessment

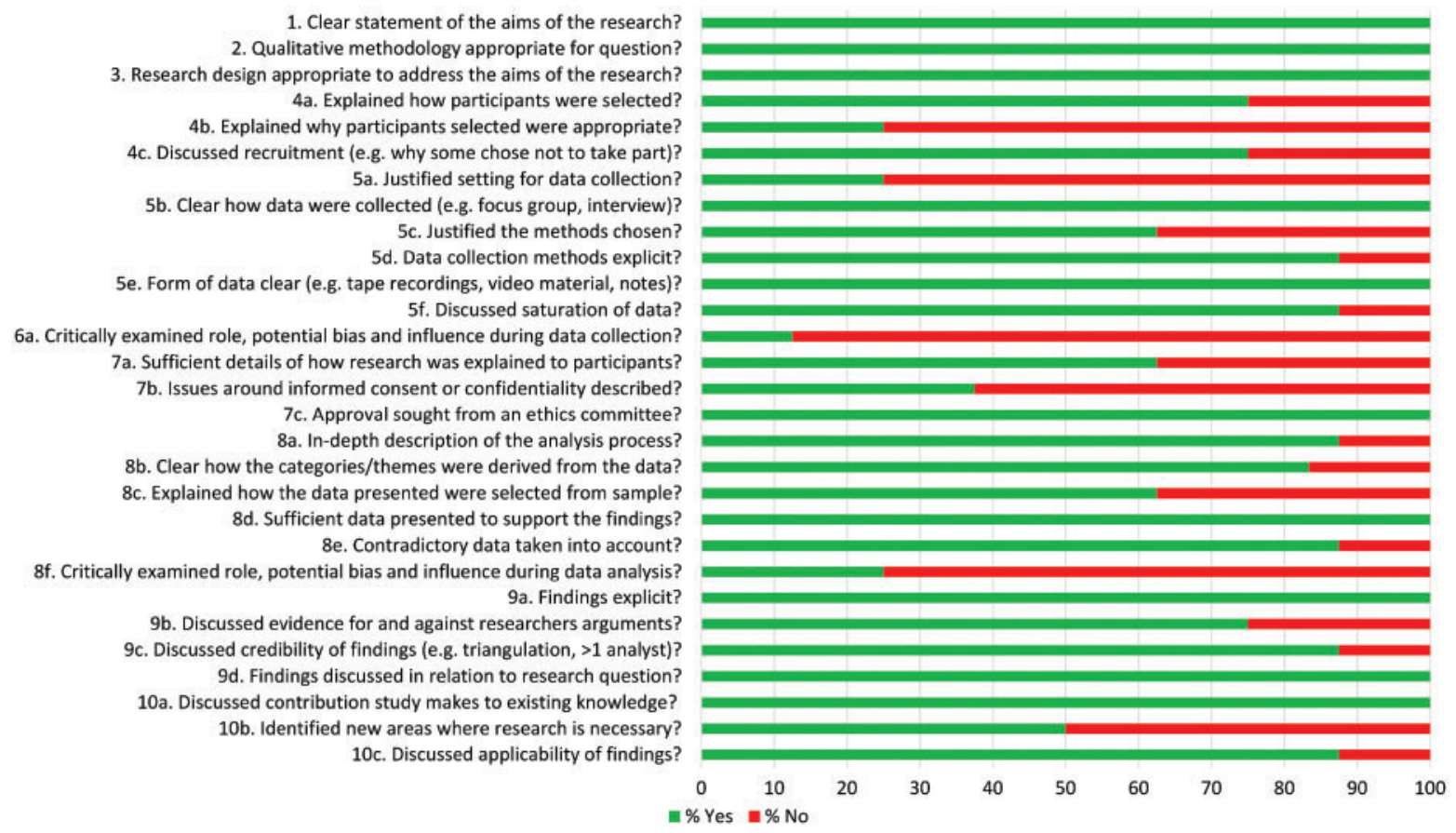

CASP: Critical Appraisal Skills Programme.

\section{Discussion}

Our synthesis suggests that patients with shoulder disorders contend with considerable disruption to their life. They can experience difficulties in performing self-care activities, work and leisure, which can cause considerable distress (particularly frustration and anxiety). Some experiences (e.g. pain, physical function/activity limitations and sleep disruption) are more common than others (e.g. cognitive dysfunction and other pathophysiological manifestations). Also, some experiences appear to impact on 
FIG. 3 Interrelationship between themes describing the experiences of people with shoulder disorders

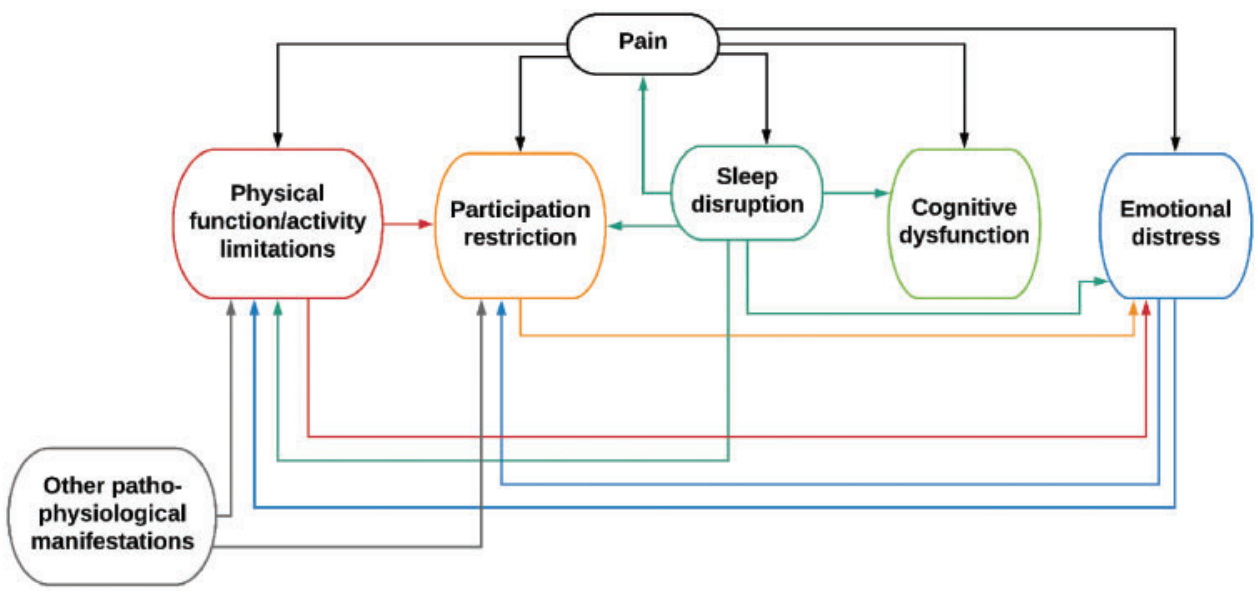

TABLE 2 GRADE-CERQual Summary of Qualitative Findings table

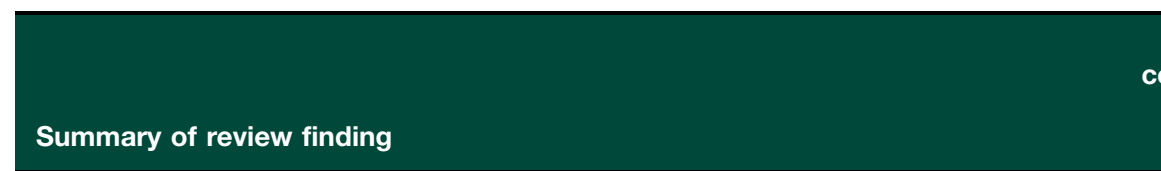

Pain

Pain was the predominant symptom experienced by people with rotator cuff disease, adhesive capsulitis, proximal humeral fracture or unspecified shoulder pain. Many people reported severe pain. Some people experienced worse pain at night or intense pain only on certain movements.

Physical function/activity limitations

Difficulty performing activities of daily living: most people with rotator cuff disease, adhesive capsulitis, proximal humeral fracture or unspecified shoulder pain reported that shoulder pain affected their ability to carry out activities of daily living. These activities include dressing, bathing/grooming, cooking, hanging washing, shopping, lifting/carrying and driving. In some cases, activity limitations were attributed to difficulties with purposeful movements (e.g. overhead use, reaching, pushing/pulling, prolonged use in one position).

Avoiding movements: many people with rotator cuff disease, adhesive capsulitis, shoulder instability or unspecified shoulder pain reported avoiding movements that aggravated the pain and changing the way they performed particular activities.

Some avoided certain movements or activities due to fear of re-injury.

Participation restriction

Work disruption: pain and difficulties with purposeful movements affected the work performance of many people with rotator cuff disease, adhesive capsulitis or unspecified shoulder pain. Some people reported experiencing problems undertaking physically demanding work tasks. A few people described problems with concentration due to pain, which negatively impacted work performance.

Limited recreation: in many people with rotator cuff disease, adhesive capsulitis, proximal humeral fracture, shoulder instability or unspecified shoulder pain, pain and difficulties with purposeful movements also affected the ability to engage in recreational/leisure activities, such as gardening and sports requiring heavier hand and arm use (e.g. tennis, climbing).

Limited social interactions: some people with rotator cuff disease or unspecified shoulder pain reported that shoulder pain restricted their social interactions (e.g. they needed someone to drive them to events, avoided crowded places due to fear of exacerbating their pain if bumped into). In some cases, interpersonal relationships were adversely affected (e.g. changed role within family).

Sleep disruption

Most people with rotator cuff disease, adhesive capsulitis, proximal humeral fracture or unspecified shoulder pain reported that pain at night disrupted their sleep. The

\section{Studies}

contributing to

the review

finding

[24-30]

[24-30]

[24-28, 30, 31]

Moderate confidence

[24-28, 30]

Moderate confidence

[24, 25, 27-31] Moderate confidence

$[27,28,30]$

Moderate confidence

Moderate confidence 


\begin{tabular}{lcc} 
& $\begin{array}{c}\text { Studies } \\
\text { CERQual } \\
\text { Summary of review finding } \\
\text { the review to } \\
\text { finding }\end{array}$ & $\begin{array}{c}\text { assessment of } \\
\text { confidence } \\
\text { in the evidence }\end{array}$ \\
\hline
\end{tabular}

type of sleep disruption varied; people either had trouble getting to sleep, were woken by pain, struggled to get back to sleep due to constant 'nagging' pain, experienced problems when lying on their affected side or experienced difficulties turning over in bed. Some reported that sleep deprivation negatively affected their cognition (e.g. concentration, memory), emotions (i.e. made them irritable), and ability to perform daily activities and participate in work. A few people perceived their shoulder pain to be aggravated by tiredness.

Cognitive dysfunction

A few people with rotator cuff disease or adhesive capsulitis reported that their shoulder pain was so severe that it prevented them from being able to concentrate on anything else. In some cases, sleep deprivation due to shoulder pain affected people's concentration and memory, which in turn affected their work performance.

\section{Emotional distress}

Frustration: many people with rotator cuff disease, adhesive capsulitis, shoulder instability or unspecified shoulder pain reported feeling frustrated and annoyed by their pain. Some people reported feeling frustrated by their inability to complete daily activities, recreational activities or physically demanding work tasks. Some people found that the sleep deprivation associated with their shoulder pain made them irritable.

Anxiety: a few people with rotator cuff disease, adhesive capsulitis, shoulder instability or unspecified shoulder pain reported feeling worried about what could be the cause of their pain. Some were fearful of certain movements, activities or social interactions that might exacerbate the pain or cause re-injury.

Depression: some people with rotator cuff disease, shoulder instability or unspecified shoulder pain reported that their pain and reduced activities made them feel depressed.

Hidden suffering: a few people with rotator cuff disease or adhesive capsulitis reported that pain and impairments in activities of daily living occurred without obvious outward signs, so that much of the suffering involved was hidden from the casual observer. This led to the perception of much less sympathy from others than would be the case with many other conditions.

Other pathophysiological manifestations

Some people with rotator cuff disease, adhesive capsulitis or unspecified shoulder pain reported other pathophysiological manifestations, including reduced range of motion, loss of strength (including lack of grip), increased muscle tension, loss of automatic protective reactions, instability and problems when weight-bearing through the affected limb. A few people attributed their difficulties with certain activities (e.g. lifting, overhead use) to poor muscle strength rather than pain.

$$
\begin{aligned}
& \text { Very low } \\
& \text { confidence }
\end{aligned}
$$
$[24,25,27,30, \quad$ Moderate 31] confidence

$\begin{array}{cc}{[26,28,30,31]} & \begin{array}{c}\text { Moderate } \\ \text { confidence }\end{array} \\ {[27,30,31]} & \begin{array}{c}\text { Moderate } \\ \text { confidence }\end{array} \\ {[26,28]} & \text { Low } \\ & \text { confidence }\end{array}$

$[24,26,27,30] \quad$ Low

confidence

Objective: to identify, appraise and synthesize qualitative research evidence on the experiences (including symptoms and perceived impacts on daily living) of people with a shoulder disorder. Perspective: experiences and perspectives of people with any shoulder disorder living in any country. ${ }^{a}$ Explanations of each CERQual assessment are provided in the evidence profile presented in supplementary Table S4, available at Rheumatology online. GRADE-CERQual: Grading of Recommendations Assessment, Development and Evaluation Confidence in Evidence from Reviews of Qualitative research; High confidence: highly likely that the review finding is a reasonable representation of the phenomenon of interest; moderate confidence: likely that the review finding is a reasonable representation of the phenomenon of interest; low confidence: possible that the review finding is a reasonable representation of the phenomenon of interest; very low confidence: unclear whether the review finding is a reasonable representation of the phenomenon of interest [19].

others (e.g. pain can lead to avoidance of particular activities and to sleep disruption), which suggests that addressing one may alleviate the other(s) in some patients. We considered it likely that most of the review findings were a reasonable representation of the experiences of people with shoulder disorders.

There are several strengths of our review. We pre-specified our methods and registered our review prior to formal screening of search results against eligibility criteria. Errors in selection, extraction, appraisal and coding of studies were minimized by the involvement of at least two authors in each process. We were able to obtain rich, unpublished data for one of the included studies [30]. We included studies regardless of the type of shoulder disorder, setting or country, which enhances the generalizability of our findings. Unlike most systematic reviews of qualitative research evidence, we formally assessed our confidence in each of the review findings using the recently developed GRADECERQual framework [19].

The review findings should be considered in light of some limitations. Our previous research to identify important outcomes for shoulder disorders [4, 5, 9] may have influenced our analysis and selection of data for presentation. We attempted to minimize this influence by having 
TABLE 3 Illustrative quotes reflecting each theme describing the experiences of people with a shoulder disorder

Theme

Pain

Physical function/activity limitations

Difficulty performing

activities of daily living

Avoiding movements

Participation restriction

Work disruption

Limited recreation

Limited social

interactions

Sleep disruption

Cognitive dysfunction

Emotional distress

Frustration

Anxiety

Depression

Hidden suffering

Other pathophysiological manifestations

\section{Illustrative quotes ${ }^{a}$}

'It was only maybe a few weeks after that I began to find my shoulder getting really, really sore ... Well, it gradually just got worse and worse. Eventually it was really extremely painful' [24].

'I have had [a] back operation, I have fallen out of trees but this pain was far worse than any other pain I have ever had in my life' [27].

'Try getting out the bath 'cause you can't find a way up. You just cannot find a way to get out the bath! We've got bars on the side of the bath anyway but I couldn't find a way. I just had to snake over the side sometimes' [26].

'Shopping was more difficult, food shopping, that sort of thing, not being able to drive or carry the stuff back' [30].

'I will have to do it with the other arm, because I don't want to hurt it (my shoulder)' [27].

'My fear of dislocating it again has made me just more apprehensive about playing ... so I stopped' [31].

'I could'na work at all well, I could'na work with my tools the way I usually did ... I had to know my limits ... It was hard to work but I had to ... It was there all the time through the day ...' [24].

'I need to work more hours and ... I physically can't' [27].

'I can't lift the [grand]children. I don't walk my dogs for fear of that (. . .) pulling' [25].

'I couldn't play tennis, absolutely out of the question, I couldn't even lift the racquet up' [27].

'It is not fun to go and sit somewhere a long time'; 'In a large crowd someone could bump into you' [28].

'It has been a big upheaval and I'm lucky that I've got some good friends to take me out nights' [30]

'At night particularly, if I lay on it and turned over it would wake me up and l'd give a little yelp of pain, annoy the wife, roll over again ... I spent the night spinning round' [24].

'... trying to find a comfortable position to sleep, terrible ... then turn over suddenly and it was painful' [27].

'In my particular case it gave really violent pain to the point of not being able to concentrate on anything else' [24].

'One night, two nights I can do, three nights I'm starting to get really tired (...) memory getting really ditsy at work' [25].

'It made me more moody and lose my temper with people ... my family definitely noticed a difference, I was more snappy ...' [24].

'[The pain] would annoy me more than anything, rather than worry me. Because I get frustrated if I can't do everything' [25].

'Even now in the bath I feel a bit anxious because you can feel a bit trapped' [30].

'I'm frightened l'm going to suddenly get that sharp pain' [30].

'It (shoulder) just wears you down ... I just got so down about it. It was awful. So she (GP) put me on amitriptyline ... it was not a good time in my life' [27].

'I remember feeling hopeless ... that my shoulder would never recover' [31].

'... you don't get any sympathy from people because it is not something that stops you doing things but in yourself you feel so frustrated, because when I reached up to do anything, we do a lot of overhead stuff, there was no strength in the arm, it was very painful but nobody would say, "How is it?" You felt that they didn't understand, because you are at work with it and that was the hardest thing, and they still expected you to do things because you didn't have your arm strapped up' [26].

'Others do not understand when it is not visible' [28]

'I would have to use the good arm to lift it, I've no strength at all in that arm, above about that high' [27].

'I just have no movement in me arm at all. I can sort of get it half way up in front of me and half way to the side of me ... but I can't raise me arm above me head' [30].

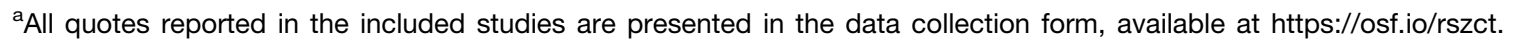

a second researcher not involved in our prior work (D.A.O.) code the studies. Also, we have uploaded our data collection and coding form to the Open Science Framework (https://osf.io/rszct), so that readers can see the text and quotes that underlie each theme generated.
Another limitation is that we were unable to assess the risk of reporting biases on our review findings, given the lack of suitable methods for qualitative evidence syntheses [44]. Finally, our findings may not reflect the experiences of people with glenohumeral or acromioclavicular OA, 
glenoid labrum pathologies or dislocation of the shoulder, given that no such people were included in the studies we identified.

It is important that clinicians ask patients which problems are arising because of their shoulder disorder and which they would like to focus on. If a mismatch exists between what clinicians and patients consider important in terms of progress and recovery, then patients may not receive the care most appropriate for them. For example, addressing strength or range of movement may not be most helpful to a patient whose main concerns might be night pain or anxiety about certain movements causing further damage, or how to modify activities so that they can continue to work. Addressing treatment expectations and supporting psychosocial needs may help improve outcomes for people with shoulder disorders.

There are several avenues for further research in this area. It would be useful to explore the experiences of people with shoulder disorders in low- and middleincome countries, as these may differ from those documented in this review, which is based on data from high-income countries only. Such research could also be conducted on people with shoulder disorders that were not represented in this review (e.g. glenohumeral OA) or on people underrepresented in this review (e.g. only $11 \%$ of participants with rotator cuff tear or instability were women). Finally, more methodological research could be conducted to evaluate the contribution of qualitative evidence syntheses to the development of core domain sets. For example, when we mapped the outcome domains arising from the current review of qualitative studies against the domains included in our 2016 preliminary core domain set for shoulder disorders [9], we uncovered one potentially missing domain-cognitive dysfunction. This and other findings of our review informed discussions at the OMERACT 2018 conference, where we sought endorsement from the wider OMERACT community on our core domain set for shoulder disorders [45].

Our thematic synthesis revealed that patients with shoulder disorders may need to contend with several disruptive experiences, including pain, physical function/activity limitations, participation restriction, sleep disruption, cognitive dysfunction, emotional distress, and other pathophysiological manifestations such as loss of muscle strength or reduced range of shoulder movement. A better understanding of patients' experiences is useful for clinicians who treat them and can also inform selection of the most appropriate outcomes to measure in clinical trials and other research studies for shoulder disorders.

\section{Acknowledgements}

We thank Carol Payne (Norfolk and Norwich University Hospital) for providing us with unpublished data from her study. M.J.P. is supported by an Australian National Health and Medical Research Council (NHMRC) Early Career Fellowship. R.B. is supported by an Australian NHMRC Senior Principal Research Fellowship. The funders had no role in study design, data collection and analysis, decision to publish, or preparation of the manuscript. All authors declare to meet the ICMJE conditions for authorship. R.B. conceived the study design. M.J.P., D.A.O., D.B., D.A.v.d.W., J.J.G. and R.B. provided input into the study design. M.J.P. and M.M. selected studies for inclusion. M.J.P., D.A.O. and M.M. collected data from studies. M.J.P., R.H. and M.M. assessed the quality of studies. M.J.P. and R.H. assessed the confidence in review findings. M.J.P. and D.A.O. performed data coding and thematic synthesis, with contribution from R.B. M.J.P. wrote the first draft of the article. All authors contributed to revisions of the article. All authors approved the final version.

Funding: No specific funding was received from any funding bodies in the public, commercial or not-for-profit sectors to carry out the work described in this manuscript.

Disclosure statement: D.B. is an author of one of the studies included in this systematic review, but was not involved in eligibility assessment, data collection or quality assessment. The other authors have declared no conflicts of interest.

\section{Data availability}

The study protocol, data collection form and coding for this study are available on the Open Science Framework: https://osf.io/vq4mz/.

\section{Supplementary data}

Supplementary data are available at Rheumatology online.

\section{References}

1 Linsell L, Dawson J, Zondervan K et al. Prevalence and incidence of adults consulting for shoulder conditions in UK primary care; patterns of diagnosis and referral. Rheumatology (Oxford) 2006;45:215-21.

2 Ostör AJ, Richards CA, Prevost AT, Speed CA, Hazleman $B L$. Diagnosis and relation to general health of shoulder disorders presenting to primary care. Rheumatology (Oxford) 2005;44:800-5.

3 Largacha M, Parsons IM IV, Campbell B et al. Deficits in shoulder function and general health associated with sixteen common shoulder diagnoses: a study of 2674 patients. J Shoulder Elbow Surg 2006;15:30-9.

4 Page MJ, Huang $H$, Verhagen AP, Buchbinder R, Gagnier JJ. Identifying a core set of outcome domains to measure in clinical trials for shoulder disorders: a modified Delphi study. RMD Open 2016;2:e000380.

5 Page MJ, Huang H, Verhagen AP, Gagnier JJ, Buchbinder $R$. Outcome reporting in randomized trials for shoulder disorders: literature review to inform the development of a core outcome set. Arthritis Care Res (Hoboken) 2018;70:252-9.

6 Gagnier JJ, Page MJ, Huang H, Verhagen AP, Buchbinder R. Creation of a core outcome set for clinical trials of people with shoulder pain: a study protocol. Trials 2017;18:336. 
7 Boers M, Kirwan JR, Wells G et al. Developing core outcome measurement sets for clinical trials: OMERACT Filter 2.0. J Clin Epidemiol 2014;67:745-53.

8 Page MJ, McKenzie JE, Green SE et al. Core domain and outcome measurement sets for shoulder pain trials are needed: systematic review of physical therapy trials. $\mathrm{J}$ Clin Epidemiol 2015;68:1270-81.

9 Buchbinder R, Page MJ, Huang $\mathrm{H}$ et al. A preliminary core domain set for clinical trials of shoulder disorders: a report from the OMERACT 2016 Shoulder Core Outcome Set Special Interest Group. J Rheumatol 2017;44:1880-3.

10 Boers M, Kirwan JR, Tugwell P et al. The OMERACT handbook, Updated 19 January 2018: OMERACT.

11 Williamson PR, Altman DG, Bagley $\mathrm{H}$ et al. The COMET handbook: version 1.0. Trials 2017;18:280.

12 Keeley T, Williamson P, Callery P et al. The use of qualitative methods to inform Delphi surveys in core outcome set development. Trials 2016;17:230.

13 Tong A, Flemming K, Mclnnes E, Oliver S, Craig J. Enhancing transparency in reporting the synthesis of qualitative research: ENTREQ. BMC Med Res Methodol 2012;12:181.

14 Whittle S, Buchbinder R. In the clinic. Rotator cuff disease. Ann Intern Med 2015;162:ITC1-15.

15 Moher D, Liberati A, Tetzlaff J, Altman DG, Group P. Preferred reporting items for systematic reviews and meta-analyses: the PRISMA statement. PLoS Med 2009;6:e1000097.

16 Critical Appraisal Skills Programme (CASP). CASP Qualitative Research Checklist: 10 Questions to Help You Make Sense of Qualitative Research. Oxford: Public Health Resource Unit; UK: Milton Keynes Primary Care Trust, 2002.

17 Slade SC, Kent P, Bucknall T et al. Barriers to primary care clinician adherence to clinical guidelines for the management of low back pain: protocol of a systematic review and meta-synthesis of qualitative studies. BMJ Open 2015;5:e007265.

18 Thomas J, Harden A. Methods for the thematic synthesis of qualitative research in systematic reviews. BMC Med Res Methodol 2008;8:45.

19 Lewin S, Bohren M, Rashidian A et al. Applying GRADECERQual to qualitative evidence synthesis findings-paper 2: how to make an overall CERQual assessment of confidence and create a Summary of Qualitative Findings table. Implement Sci 2018;13:10.

20 Munthe-Kaas H, Bohren MA, Glenton C et al. Applying GRADE-CERQual to qualitative evidence synthesis findings-paper 3: how to assess methodological limitations. Implement Sci 2018;13:9.

21 Colvin CJ, Garside R, Wainwright $M$ et al. Applying GRADE-CERQual to qualitative evidence synthesis findings-paper 4: how to assess coherence. Implement Sci 2018;13:13.

22 Glenton C, Carlsen B, Lewin S et al. Applying GRADECERQual to qualitative evidence synthesis findings-paper 5: how to assess adequacy of data. Implement Sci 2018;13:14.
23 Noyes J, Booth A, Lewin S et al. Applying GRADECERQual to qualitative evidence synthesis findings-paper 6: how to assess relevance of the data. Implement Sci 2018;13:4.

24 Carter B. Clients' experiences of frozen shoulder and its treatment with Bowen technique. Complement Ther Nurs Midwifery 2002;8:204-10.

25 Gillespie MA, Cznik MA, Wassinger CA, Sole G. Rotator cuff-related pain: patients' understanding and experiences. Musculoskelet Sci Pract 2017;30:64-71.

26 Jones S, Hanchard N, Hamilton S, Rangan A. A qualitative study of patients' perceptions and priorities when living with primary frozen shoulder. BMJ Open 2013;3:e003452.

27 Minns Lowe CJ, Moser J, Barker K. Living with a symptomatic rotator cuff tear 'bad days, bad nights': a qualitative study. BMC Musculoskelet Disord 2014;15:228.

28 Nyman P, Palenius K, Panula H, Mälkiä E. Patients' experiences of shoulder problems prior to and following intervention. Physiother Theory Pract 2012;28:221-31.

29 O'Hara NN, Garibaldi A, Sprague S et al. Rehabilitation, not injury or treatment details, dominate proximal humeral fracture patient concerns: a thematic analysis. Eur J Pers Cent Healthc 2017;5:351-6.

30 Payne $\mathrm{C}$. The assessment of outcome for musculoskeletal shoulder pain: a mixed methods study. Norwich, UK: University of Anglia, 2010.

31 Tjong VK, Devitt BM, Murnaghan ML, Ogilvie-Harris DJ, Theodoropoulos JS. A qualitative investigation of return to sport after arthroscopic bankart repair: beyond stability. Am J Sports Med 2015;43:2005-11.

32 Bernhardsson S, Larsson MEH, Johansson K, Öberg B. "In the physio we trust": a qualitative study on patients' preferences for physiotherapy. Physiother Theory Pract 2017;33:535-49.

33 Calner T, Isaksson G, Michaelson P. "I know what I want but I'm not sure how to get it"-expectations of physiotherapy treatment of persons with persistent pain. Physiother Theory Pract 2017;33:198-205.

34 Eriksson L, Lindström B, Ekenberg L. Patients' experiences of telerehabilitation at home after shoulder joint replacement. J Telemed Telecare 2011;17:25-30.

35 Harding G, Parsons S, Rahman A, Underwood M. "It struck me that they didn't understand pain": the specialist pain clinic experience of patients with chronic musculoskeletal pain. Arthritis Rheum 2005;53:691-6.

36 Hunhammar C, Nilsson-Wikmar L, Löfgren M. Striving to master variable pain: an interview study in primary care patients with non-specific long-term neck/shoulder pain. J Rehabil Med 2009;41:768-74.

37 Hutting N, Heerkens YF, Engels JA, Staal JB, Nijhuis-van der Sanden MW. Experiences of employees with arm, neck or shoulder complaints: a focus group study. BMC Musculoskelet Disord 2014;15:141.

38 Hutting N, Detaille SI, Heerkens YF et al. Experiences of participants in a self-management program for employees with complaints of the arm, neck or shoulder (CANS): a mixed methods study. J Occup Rehabil 2017;27:35-48. 
39 Wiitavaara B, Brulin C, Barnekow-Bergkvist M. When the body makes itself heard-the experience of bodily illness among people with neck-shoulder problems. Adv Physiother 2008;10:85-94.

40 Littlewood C, Malliaras P, Mawson S, May S, Walters S. Patients with rotator cuff tendinopathy can successfully self-manage, but with certain caveats: a qualitative study. Physiotherapy 2014;100:80-5.

41 Cuff $A$, Littlewood C. Subacromial impingement syndrome-what does this mean to and for the patient? A qualitative study. Musculoskelet Sci Pract 2018;33:24-8.

42 Sandford FM, Sanders TAB, Lewis JS. Exploring experiences, barriers, and enablers to home- and class-based exercise in rotator cuff tendinopathy: a qualitative study. J Hand Ther 2017;30:193-9.

43 Sundberg G. Patients' experiences of the rehabilitation period and long-term effects of rehabilitation after rotator cuff repair. Nordisk Fysioterapi 2004;8:183-9.

44 Booth A, Lewin S, Glenton C et al. Applying GRADECERQual to qualitative evidence synthesis findings-paper 7: understanding the potential impacts of dissemination bias. Implement Sci 2018;13:12.

45 Ramiro S, Page MJ, Whittle SL et al. The OMERACT core domain set for clinical trials of shoulder disorders $\mathrm{J}$ Rheumatol 2019. doi:10.3899/jrheum.181070. 